\title{
Study on Content and Organization of Mathematical Cognitive Structure in Mainland China
}

\author{
Dandan Sun, Zezhong Yang* \\ School of Mathematics Science, Shandong Normal University, Jinan, China \\ School of Mathematics Science, Shandong Normal University, Jinan, China \\ Email:zhongzee@163.com
}

Keywords: Mathematical Cognitive Structure; Content; Organization; Presentation form

\begin{abstract}
This paper focuses on the studies on mathematical cognitive structure in mainland China, summarizes and evaluates current studies through classifying them into three parts: the contents, the presentation and the organization.
\end{abstract}

\section{Introduction}

Mathematical cognitive structure is an internal psychological structure formed during the mathematics learning process. Researches and practices over the years about mathematical education show that cognitive structure of mathematics has a very important role in learning mathematics for students. Thus, researchers attach particular importance to the study of mathematical cognitive structure, especially the study about content and organization of mathematical cognitive structure, because study on this aspect can help people to find the optimal mathematical cognitive structure and improve the quality of teaching mathematics directly.In order to make the current studies go further, this paper will review and summarize them.

\section{The content of mathematical cognitive structure}

Cao Caihan and Cai Jinfa deem that the mathematical cognitive structure is mainly composed of mathematical knowledge which is constructed by the students. Specifically, it includes basic mathematical concept, terminology, theorem and laws, and knowledge about method of mathematical activities, and knowledge of mathematical history and mathematical evaluation[1].

Wang Guangming and Wang yue think the content of mathematical cognitive structure mainly includes the network links of mathematical concepts, the relation of mathematical propositions, operation systems of mathematical skills and mathematical thought and methods[2].

$\mathrm{Xu}$ wensheng and Cui junying believe that the mathematical cognitive structure includes mathematical theories and skills, experience of mathematical activities. The experience has two parts, the first one is the summary of background and conditions in which specific mathematical thought and methods used, the other one is summary of general ways in mathematical activities [3].

He Xiaoya thinks the content of mathematical cognitive structure has three different concepts. The first one is basic concepts, it is mainly include verbal information or image information and is

* Yang zezhong, Professor, Research area: mathematical education and history. Corresponding author. Email: zhongzee@163.com 
obtained by students through studying some mathematical concepts and propositions . The second one is the conception of specific mathematical methods, it is formed in the process of solving problems .The last one is the concept of the strategy of solving mathematical problems [4].

Liu bin thinks the mathematical cognitive structure includes three types of knowled ge. The first is general knowledge such as concept and theorem. The second is the middle level knowledge, such as the way to solve problem. The third is a high level of knowledge such as mathematical methodology and views of mathematics[5].

Zhang Jiemin believes that the mathematical cognitive structure includes various of internalized mathematical theories, skills and conceptions [6].

Guan peng thinks that the mathematical cognitive structure is an organic combination which is made up of perceptual and rational knowledge of mathematics, students' individual unique knowledge of mathematics and the cognitive operating system[7].

Long Yi considers that mathematical cognitive structure should contain three major components: the first is knowledge and experience system of mathematics; the second is the operating system of mathematical cognitive activities, the third is the meta-cognitive system of mathematics. Long Yi believes that knowledge and experience system of mathematics is mainly internalized basic knowledge and skills of mathematics; Operating system includes attention, perception, memory, image, and thought in mathematical cognitive activities, while meta-cognitive system includes meta-cognitive knowledge, experience, monitoring and regulation concerning mathematical activity[8].

$\mathrm{Yu}$ Ping points out that the mathematical cognitive structure is mainly an individual representation of various kinds of mathematical knowledge. The content of mathematical cognitive structure consists of three types: declarative knowledge, procedural knowledge and process knowledge. Among them, the procedural knowledge can be divided into intellectual skills and cognitive strategies. Intellectual skills are composed of simple and complex operation skills. At the same time, cognitive strategies can be further divided into strategic knowled ge and meta-knowledge. Process knowledge refers to the experiential knowledge obtained during mathematical activities [9].

Li Shiqi points out that, besides mathematical knowledge, mathematical cognitive structure also contains knowledge of other aspects, like knowledge of language, physics, chemistry, geography, astronomy, etc. It even includes meta-knowledge [10].

\section{The presentation form of knowledge in mathematical cognitive structure}

Guan Peng and Chen Jie believe that knowledge in mathematical cognitive structure is mainly summarized in the semantic manner, and it generally exists in the minds in the form of intuition [7] [11].

He Xiaoya points out that the presentation form of procedural knowledge is "production", which includes three types: forward, reverse and transformable production [4].

Yu Ping pointed out that the representation of declarative knowled ge of mathematics is a mutual intertwine and integration of propositional network and image representation. The image of mathematical concept is not only a sensual image formed on the basis of the perception of things , but more a case of "imaginary representation" or "representation of instances ", which individuals construct an model can express object through their own imagination. In order to make the mathematical concept precise, it must be characterized by the propositional network. Procedural knowledge is represented by dynamic "production". The so-called production is an rule of "if ...... 
then ......", that is to say, a production is a program that an act occurs when certain conditions meet. Characterization of procedural knowledge of mathematics is divided into two levels, the first is characterization of relationship, the second is the ideal characterization. Characterization of relationship is the individual realization of relationship existed between the knowledge during its development process. Ideal characterization is the realization of the reason why there are some relationships between the knowledge, the more component is a meta-cognitive experience [9].

\section{The organization of knowledge in mathematical cognitive structure}

Cao Caihan considers that there is a hierarchically logical link between the whole and the parts of mathematical knowledge, the apex of the level is the most subsumed concepts and propositions, the following are specific knowledge such as concept and proposition within a lesser subsumed scope [12].

He Xiaoya, Liu Bin, Chen Jie and Zhong Yuanchu holds that the internal concepts of mathematical cognitive structure forms lots of knowledge chunks of all sizes which are shaped like "pyramid". And then these small "pyramid" structure interrelated to be a three-dimensional network structure which is hierarchical and clear. With the process of math learning, knowledge chunks in the mathematical cognitive structure is further organized, classified and summarized, along with constantly enrichment of the internal and further reorganization of the external, but in terms of the whole structure, it is still a "pyramid" structure "grid" type [4,5,11,13].

Chen Jie, Zhong Yuanchu, ,Ma Yue insists that there are no rigid logical order between the content of the mathematical cognitive structure in the brains of all the students. It is neither a coherent linear structure, nor an well-ordered network structure. Compared with the mathematical knowledge structure, the logic sequence and hierarchy between its content always be weakened. Guan Peng thinks that it is structured by another form, both cumulative and hierarchical, so it is both a hierarchical and network structure [11,13,14].

Yu Ping and Shan Zun proposed a cognitive structure possessing mathematical characteristics: structure of CPFS. It is called CPFS structure made of concept field, concept system, proposition field, proposition system. The meanings of the CPFS structure are: (1) the mathematical knowledge network which is internalized in the individual mind. There are equivalent abstract relationship, strong abstract relationship, weak abstract relevance, and generalized abstract relevance among the knowledge. (2)It is with certain abstract relationship which itself contains ways of thinking between knowledge in the networks that the network link between the various knowledge contains mathematical methods, namely "Connection set" as a "methodological system." As the mathematical knowledge, logical equivalence, strong abstraction, weak abstraction, and generalized abstraction, and others, are the unique relationship between mathematical concepts and the propositions. Thus, CPFS theory accurately describes the organization of mathematical knowledge in the individual mind [15].

Li Shiqi thinks mathematical cognitive structure can be regarded as a complex network composed of nodes and on-line in form. The network has three basic forms: linear structure, tree structure and network architecture. They can also be divided into two hierarchical categories: horizontal and vertical relationship by the particular relationship between knowledge nodes . The knowledge of mathematical cognitive structure can be combined with each other in three forms, vertical and horizontal structures can also intersect or overlap with each other, a connection may emerge between two separate structures via establishing on-line between a few nodes. Structure is usually made by 
more basic structures. That is to say, each node exists as block. itself comprises a plurality of nodes, forming a structure. It may be a linear structure, a tree structure or a network structure and it may contain longitudinal hierarchy or lateral web-like construction[10].

\section{Conclusions}

From above studies, it can be found that it is generally accepted that mathematical cognitive structure is a psychological form of mathematical knowledge that is internalized. The content of mathematics cognitive structure includes mathematical concepts, mathematical propositions, mathematical thought and methods and so on. These contents have regular connections with each other. The views about representations of knowledge in mathematical cognitive structure have not been unified yet.The widely accepted view is that the knowledge in cognitive structure of mathematics is characterized in two ways, verbal system and image system. Some scholars think of the representation system of mathematical knowledge from a different angle, corresponding with classifications of knowledge in mathematical cognitive structure. The opinions about organizational forms of mathematical knowledge experienced such a progress: firstly, researchers emphasize the upper and lower levels of the knowledge, afterwards, they emphasize the horizontal linkages of knowledge, they believe that the knowledge contacts with each other to construct a complex three-dimensional network.

By reviewing above studies, it is found there are some shortcomings at present also, such as (1) the deduction studies are obviously more than empirical studies. (2) Many studies results come from common educational psychology, not directly come from mathematical teaching or learning. (3) There is not a quantitative study on cognitive structure of mathematics yet. So the wider and deeper studies on mathematical cognitive structure are necessary.

\section{References}

[1] Cao C. H.\& Cai J. F., Introduction to Mathematics Education, Nanjing, Jiangsu Education Press, 1989.

[2] Wang G. M.\& Wang Y., The comparison of the mathematical cognitive structure between the top and ordinary student in senior high school, the possible reason and teaching suggestions, Preference for Middle School Mathematical Education , 12(2004) 1-4.

[3]Xu W. S.\& Cui J.Y., Some Discussion on Mathematical Realization Structure, Journal of Hebei Normal University (Natural Science), 04(1993) 24-27.

[4]He X. Y., Instructional Strategies on Constructing well Mathematical Cognitive Structure, Journal Of Mathematics Education, 11 (2002) 24-27.

[5]Liu B., The Structure of Mathematics Recognition and Its Building, Journal of Hubei University (Natural Science), 19(1997) 222-226.

[6]Zhang J. M., Consideration about the theory and practice of the development and improvement of student cognitive structure, Mathematics Bulletin, 8(1996) 16-20.

[7]Guan P., The cognitive psychology principles about how to form good cognitive structure of mathematics , Educational Theory and Practice, 18(1998) 40-45. 
[8]Long Y., A Discussion on Mathematical Cognitive Structure, Journal of Jishou University (Natural Science edition), 17(1996) 28-31.

[9]Yu P., Psychology of Mathematics Education, Nanning, Guangxi Education Publishing House, 2004.

[10] Li S. Q., PME: Psychology of Mathematics Education, Shanghai, East China Normal University Press, 2001.

[11]Chen J., The study of optimizing and perfecting mathematics cognition structures of mid-school class teaching, Jinan, Shandong Normal University, (2003) 2-30.

[12] Cao C. H \& Zhang J. Y. Psychology of Mathematics Education, Beijing, Beijing Normal University Press, 1999.

[13]Zhong Y. C., On Improving the Systemization of High-school Students' Mathematical

Cognitive Structure ,Changsha, Hunan Normal University, (2006) 5-20.

[14]Ma Y., Some Discussion on Mathematical Cognitive Structure, World of Mathematics teaching guide, 03(2012) 17-18.

[15]Yu P. \& Shan Z., Theory of CPFS Frame in Mathematics Learning, Journal Of Mathematics Education, 12(2003) 12-16. 